\title{
El uso de las Web 2.0 \\ de colaboración en los \\ escenarios virtuales de \\ divulgación científica \\ del Proyecto Atlas
}

Elias Said-Hung *

Artículo recibido:

20 de enero de 2012.

Artículo aceptado:

9 de abril de 2012.

\section{RESUMEN}

Este artículo estudia 110 enlaces vinculados al portal principal del proyecto Atlas, con el fin de analizar los rasgos generales que caracterizan el uso de las Web 2.0 de colaboración en los escenarios virtuales de divulgación científica, al interior de este estudio de caso. Los resultados apuntan, entre otras cosas, a que los escenarios estudiados se caracterizan por un bajo nivel de visibilidad en Internet, una política de acceso libre ampliamente apropiada desde esta comunidad científica, pero que tiene un bajo nivel de implementación de modelos de comunicación multiformatos a través de las Web 2.0, en los que se exponen contenidos altamente especializados, dirigidos a la exposición de

* Universidad del Norte, Colombia. saide@uninorte.edu.co.

INVESTIGACIÓN BIBLIOTECOLÓGICA, Vol. 26, Núm. 56, enero/abril, 2012, México, ISSN: 0187-358X. pp. 137-157 
informes y resultados, bajo un marco de baja apropiación de herramientas de interacción y personalización de la información. Se concluye que la presencia de un escenario virtual analizado aquí presenta limitaciones en relación con la usabilidad de las Web 2.0, en tanto que limita el ejercicio de esta función a expertos pertenecientes al mismo colectivo científico relacionado con el caso de estudio.

Palabras clave: Divulgación; Ciencia; Atlas; Escenarios virtuales; Web 2.0.

\section{ABSTRACT}

The Use of Web 2.0 for Collaboration in the Virtual Scenarios of Scientific Dissemination of the Atlas Project

Elias Said-Hung

This article analyzes 110 links related to the Atlas Project's main website, in order to study the general features that characterize the use of Web 2.0 in the virtual scenarios of scientific dissemination, within this case study. The results suggest, among other things, that the dissemination of science is characterized by a low level of visibility on the Internet, an open access policy broadly appropriate by the scientific community, but with low implementation of multi-format communication models through Web 2.0, in which highly specialized contents are exposed, aimed to the presentation of reports and results, presenting low appropriation of tools for interaction and personalization of information. It is concluded that the presence of a virtual scenario discussed here presents limitations in relation to the usability of the Web 2.0 as it limits the exercise of this function to experts belonging to the same scientific collective connected with the case study.

Keywords: Dissemination; Science; Atlas; Virtual Scenarios; Web 2.0. 


\section{INTRODUCCIÓN}

- $\mathrm{n}$ la actualidad las comunidades científicas cuentan con nuevas herra-

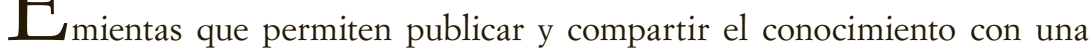
gran ventaja sobre las herramientas analógicas que se usaban con anterioridad (Biro, 2007), lo cual sugiere la necesidad de ir creando nuevos mecanismos de análisis que cubran los métodos y estrategias usadas por estas instancias académicas y/o científicas para la comunicación, difusión y divulgación del conocimiento generado en su interior.

Al momento de hacer una aproximación inicial en torno a la divulgación científica, podemos señalar que no existe una única definición sobre lo que esta labor encierra. A pesar del amplio contexto de debate alrededor de lo que significa la divulgación científica, ésta será entendida aquí como la actividad por medio de la cual se publica y propaga el conocimiento científico a un público distinto de los científicos o expertos en la materia objeto de la comunicación, (Fernández, 1999). En otras palabras, el acto de divulgación científica significa la acción de traducir o reformular un mensaje para una nueva audiencia.

Si partimos de lo hasta ahora expuesto, resulta cada vez más importante comprender los criterios y estrategias en los que es aplicada la divulgación científica al interior de la comunidad científica. Para tal fin tomamos en consideración para su estudio a la comunidad científica vinculada con el proyecto Atlas, ${ }^{1}$ una de las entidades más grandes e importantes en el campo de la física de partículas, al constar con una extensa red de investigación integrada por 3.000 miembros entre los que se encuentran 1.850 físicos diseminados en 150 instituciones ubicadas en 34 países, (U.S Departament of Energy, s.f.).

Una de las principales herramientas empleadas por el proyecto Atlas es su portal, ${ }^{2}$ integrado por una extensa diversidad de portales web articulados entre sí, destinados al trabajo colaborativo para desarrollar experimentos, intercambiar datos y revisar resultados entre los miembros de esta comunidad, entre otros aspectos; así como a la divulgación científica a partir de las publicaciones, revistas, notas y documentaciones vinculadas con este proyecto.

La compleja diversidad de espacios y recursos humanos dispuestos para los diferentes roles relacionados con las labores científicas desarrolladas desde Atlas, así como el nivel de arraigo que ésta tiene al interior de la comunidad científica vinculada a ella (física), la convierten en un caso de estudio por demás interesante que contribuye a analizar los rasgos generales que caracterizan los escenarios de divulgación, mediados por Internet, al interior

1 http://atlas.web.cern.ch/Atlas/Collaboration/

2 Ibid. 
de comunidades científicas tan complejas; en especial, en lo que se refiere a saber más acerca de los rasgos generales que caracterizan el uso de las Web 2.0 de colaboración en los escenarios virtuales de divulgación científica: las relaciones institucionales que se construyen alrededor de la divulgación; los niveles de visibilidad, popularidad y accesibilidad de la información expuesta para dicha labor; los niveles de uso de herramientas virtuales (Web 2.0), y multiformatos que ayuden a la aplicación de las estrategias de divulgación empleadas; así como la dirección hacia donde fluye esta divulgación, en lo que se refiere al tipo de público al que se dirige.

\section{LA COMUNICACIÓN Y LA DIVULGACIÓN CIENTÍ́FICA}

La ciencia debe ser comprendida como una actividad dirigida a la generación de un conjunto de códigos que ayudan a ampliar el número de aspectos vinculados con la representación de la realidad, a través de la articulación de significados y de representaciones, relacionadas con la acción académica involucrada en ella (Rubio, 1998). Es alrededor de esta labor en donde la comunicación juega un importante rol en tanto que genera un conjunto de mecanismos que contribuyen a la construcción del conocimiento científico, y la generación continua de material codificado, dirigido a comunidades no generadoras de estos saberes.

Internet y el surgimiento de redes y comunidades digitales de investigación plantean nuevos escenarios para la divulgación científica, y aunque éstos guardan cierta relación con sus homólogos analógicos, no se puede considerar que sean evaluados bajo los mismos criterios, ya que los parámetros bajo los cuales está diseminada su información son completamente ajenos a los tradicionales, (Lugo, 2004).

Es alrededor de los procesos de generación del conocimiento científico donde la comunicación formal e informal se encuentran experimentando alteraciones, ante el creciente avance de las TIC y la inclusión de éstas a favor de la difusión y la necesidad de compartir resultados científicos y académicos, para la validez de los mismos, (Russel, 2001). Lo antes señalado ha incidido en la construcción y difusión de la ciencia, desde la cada vez más compleja trama de recursos tecnológicos dispuestos para tal fin (Gómez y Arias, 2002); que aumentan así el ciclo de generación de nuevos conocimientos provenientes del aprovechamiento de programas de libre acceso que puedan ayudar a sacar máximo provecho de la visibilidad, producción intelectual y actividad académica, al interior de los grupos científicos, (Pila, 2009).

La creciente pérdida de los márgenes de acción comunicativa formales e informales alrededor de la ciencia, no sólo nos está exponiendo a un marco 
de transformación del modelo de comunicación científica, caracterizado por un nuevo entorno colaborativo, afín a lo propuesto por Glasner (1996), y de diseminación de los diferentes contenidos, al interior de los grupos o comunidades científicas, sino también a auge de nuevos mecanismos de participación y divulgación científica vinculados alrededor de los contenidos difundidos desde la web, (Harnard, 2001).

Si bien es cierto que al momento de abordar una definición unificada en torno a la divulgación científica, lo planteado en este artículo se basa en una aproximación conceptual de esta actividad, de acuerdo con lo expuesto por Fernández (1999), dicha actividad debe ser entendida como la actividad de publicación y promoción del conocimiento científico hacia una nueva audiencia.

A partir de lo expuesto por autores como Fernández (1999) o León (1999), existen diferentes tipos de escenarios de divulgación científica:

- Divulgación cientifica de expertos para expertos, donde se enmarcan aquellas piezas elaboradas dentro de un contexto en el que la audiencia hace parte del mismo colectivo científico-investigador vinculado a la disciplina del conocimiento abordado.

- Divulgación científica de expertos hacia el público en general, caracterizada por un mayor nivel de accesibilidad a personas no vinculadas con las disciplinas generadoras del conocimiento.

- Divulgación científica experto-público, representada por medio de contenidos empleados como base para los traductores del conocimiento científico, llevando éste a un lenguaje más general.

- Divulgación intermediarios-público, en el que el proceso de traducción científica toma como base otros documentos, previamente decodificados.

Sin importar el tipo de divulgación científica que se aborde, el fin último de toda comunicación alrededor de la ciencia, es que el último eslabón de la cadena de acceso al conocimiento sea un público ajeno a éste, y capaz de apropiarse significativamente de él (Estrada, 2003), y llevarla al público a un nivel en el que pueda hacer ese conocimiento parte de su vida. Es en torno a esta labor, donde los avances tecnológicos han venido adquiriendo un peso cada vez mayor, en el acceso al conocimiento, a través de la computabilidad, virtualidad, y la capacidad que brindan las Tecnologías de Información y Conocimiento (TIC), al abrir el abanico de disponibilidad y el ahorro potencial que supone la producción intelectual, donde otros aspectos deben ser tenidos en cuenta durante el proceso de divulgación científica mediado por los avances digitales. 
Con el auge de las TIC se han abierto en el campo científico nuevos tipos de herramientas y producción de documentos que contribuyen a la transmisión del conocimiento científico, y encontramos en la actualidad, no sólo diferentes tipos de publicaciones y artículos "en línea” (López y Cordero, 2005), sino también, la apertura de nuevos portales dedicados a la indexación y visibilidad de esta labor académico-científica.

Una de las principales herramientas que han contribuido a la dinamización y auge de nuevas posibilidades de acción científica a través de las TIC, son las denominadas Web 2.0. Término acuñado por O'Reilly (2005), quien da cuenta del proceso de transición de las webs tradicionales, en donde el usuario es un mero consumidor de lo que se expone en ellas, a otras más dinámicas, en las que los usuarios adquieren roles de co-editores de los contenidos generados en dichos portales.

El paso hacia una web convertida en espacio social de acceso a una información, comunicación y conocimiento cada vez más colaborativos, trae consigo el auge de una nueva generación de super-usuarios (Fumero y Roca, 2007), caracterizados por una alta capacidad de interacción ejercida desde las nuevas generaciones de navegadores, interfaces y recursos dispuestos desde la web, los cuales facilitan la creación y gestión de contenidos de forma cada vez más descentralizada y colaborativa.

La transformación del hábitat propio de la Web 1.0 a las Web 2.0 ha traído consigo la creciente entrada de una nueva etapa o fase en la que el usuario se convierte en rey, al adquirir un gran protagonismo. Ello, al pasar de ser un mero espectador y consumidor de lo expuesto en internet, a creador y generador de los contenidos y los servicios a los que éstos mismos tienen acceso, (Nafría, 2007). La transformación vivida en la nueva generación de usuarios, es resultado de la Web 2.0 y que permite la navegación colaborativa con otros miembros de este colectivo, que ayudan a crear comunidades; convirtiéndose así en el prosumidor al que aludía Toffler (1980), marcado por su capacidad de ejercer el rol de productor y consumidor de contenidos; el predominio de la horizontalidad y el trabajo colaborativo; en tanto que se facilita la presencia de usuarios anónimos que impulsan, desarrollan y gestionan proyectos, desde las redes sociales, de diferentes naturalezas, temáticas y alcances, (Tejedor et al, 2011).

Las oportunidades que trae consigo el auge de las Web 2.0 en la actualidad, abre un contexto en el campo de la educación y la ciencia, siguiendo lo expuesto por Castaño et al (2008) y Tejedor et al (2011), en donde la producción individual de contenidos; el fomento de una mayor posibilidad de 
generación de escenarios de aprendizaje con y de otros usuarios; el fortalecimiento de la cultura de la participación más activa de los usuarios, desde el aprovechamiento de herramientas cada vez más sencillas e intuitivas; la apertura de estándares no propietarios (Open Access); la creación de comunidades de aprendizaje, y el trabajo cooperativo entre iguales, entre otros aspectos, facilitan la presencia de nuevas oportunidades:

- El aprendizaje colaborativo.

- La aplicación de prácticas de validación, los contrastes y el filtrado de datos y contenidos dispuestos desde internet.

- La reproducción de escenarios reales, en donde los miembros de una comunidad virtual asumen roles de responsabilidad de la producción de la información y de contenidos online.

- La generación de nuevos contextos de reflexión, experimentación e innovación.

Por lo expuesto hasta ahora, los escenarios de divulgación científica están experimentando en la actualidad un proceso de transformación, ante la apertura de contextos virtuales orientados al ejercicio de dicha labor, y el fomento de una cultura colaborativa y de participación a nivel académico desde Internet. Por tanto, el uso del término de escenario virtual relacionado con la divulgación y la colaboración científica, además de llevar implícito el proceso vinculado con el paso de la Web 1.0 a la Web 2.0, lleva consigo: una interiorización de la cultura de la participación desde la nueva generación de webs, en la que los usuarios, miembros o no, pueden interactuar de forma dialógica, a través de los recursos de navegación y servicios dispuestos en éstos; transformando así las modalidades y las dinámicas de trabajo, así como el uso de los métodos aplicados para la generación y validación de los resultados, y los conocimientos empleados y generados para tales fines. Se trata de una nueva forma de hacer ciencia, mediada por los avances TIC: e-ciencia, caracterizada por un ejercicio más dinámico y en tiempo real del proceso de construcción de conocimientos científicos, apoyados por las redes sociales, wikis, blogs, Grid computing 3 y el cloud computing, ${ }^{4}$ entre otros recursos geográficamente distribuidos e interconectados mediante Internet.

3 Computación en red que permite el trabajo colaborativo entre instituciones y/o empresas para la organización y gestión de un conjunto de información compartida desde Internet y la interconexión de varios ordenadores ubicados geográficamente desde distintos puntos geográficos, dispuestos para el desarrollo de la labor requerida para el aprovechamiento de dicha información.

4 Es un servicio que permite el resguardo de información en una nube de cómputos, actuando como una especie de servidor desde Internet. 
La promoción de la e-ciencia traería consigo la apertura de un nuevo contexto, en el que la falsa Hipótesis de Ortega propuesto por Cole y Cole (Agenjo, 2002), y retomada por Bailón-Moreno et al, (2007) y Cabeza-Clavijo et al (2009), en las que el progreso científico estaría siendo soportado por aportes mínimos de una multitud de científicos, pudiese estar convirtiéndose en realidad, gracias a la promoción de diferentes tipos de aplicabilidad científica mediada por las TIC, de acuerdo con David (2006):

- E-ciencia orientada a la formación de comunidades, en las que su preocupación se orientaría a generar iniciativas que ayuden al diálogo sincrónico académico (Ejemplo, las iniciativas de encuentros virtuales de redes académicas nacionales y regionales como la Red Clara en América Latina). ${ }^{5}$

- E-ciencia orientada a la explotación de datos que promueven la recopilación, manejo y procesamientos de datos pertenecientes a diversos investigadores, (ejemplo, el proyecto LAMP de Princeton ${ }^{6}$ y el Proyecto Atlas estudiado aquí).

- E-ciencia orientada a maximizar los recursos tecnológicos para desarrollar mecanismos de ejecución sobre procedimientos comunes de trabajo (ejemplo, las iniciativas de Grid clusters aplicadas en el caso del estudio, Proyecto Atlas).

- E-ciencia orientada a aumentar la interactividad en el campo de la gestión, visualización y control de instrumentos entre diversos actores académicos articulados en un mismo proyecto.

Los diferentes tipos de e-ciencia, no se contraponen entre sí, sino que pueden articularse a diferentes niveles dentro de los contextos o escenarios virtuales de divulgación y colaboración científica. En la actualidad, se pueden identificar una serie de rasgos que distinguen la divulgación y la colaboración científica digital: 1) la capacidad de inclusión de elementos que contribuyen a profundizar en la información por medio de los hipertextos; lo que permite eliminar la linealidad en la lectura y facilitar el acceso a la información al conectar ideas jerarquizadas y organizadas con el fin de establecer relaciones directas e indirectas entre todos los conceptos abordados; 2) el hecho de guardar los mismos criterios que los escenarios de divulgación tradicional, en cuanto a su objetivo general, éstos se distinguen, según López y Cordero (2005), por su formato de presentación (en disco, en línea, en 
red), el tipo de acceso (acceso directo desde Internet, acceso indirecto por medio de bases de datos), la presentación de la información (completa, parcial, resumida), la fecha de publicación (con o sin periodicidad) y el estatus o condición comercial; 3) el auge de las herramientas Web 2.0, posibilita la posibilidad de compartir y difundir el conocimiento científico generado al interior de un determinado grupo de investigación, a través de escenarios no investigativos. Ello, permite, al menos potencialmente, el fortalecimiento del sistema de gestión del conocimiento, por medio del empleo de los recursos TIC y el aprovechamiento de las web 2.0, dentro de los portales web dispuestos en las diferentes comunidades científicas digitales.

Lo antes expuesto, pudiese traer consigo, como bien destacan Martínez, Peñalver y Salamanca (2007):

- La creación, desarrollo, transmisión y crítica de la ciencia, de la técnica y de la cultura.

- La preparación para el ejercicio de actividades profesionales que exijan la aplicación de conocimientos y métodos científicos y para la creación artística.

- La difusión, la valoración y la transferencia del conocimiento al servicio de la cultura, de la calidad de vida, y del desarrollo económico.

- La difusión del conocimiento y la cultura a través de la extensión universitaria/investigativa, y la formación a lo largo de la vida.

\section{Metodología}

Ante la complejidad que brinda una comunidad con la complejidad del proyecto Atlas, a partir del estudio realizado, de corte cual-cuantitativo, se tomaron como referencia un conjunto de variables propuestas por Codina (2006; 2009) y Said-Hung, Arcila-Calderón y Méndez-Barraza (2011), además de otras consideradas para el tema aquí propuesto.

Entre las variables consideradas para el abordaje de este tema tomamos la relación institucional con el proyecto Atlas, realizado por el Cern en Suiza; ${ }^{7}$ el tipo de enlace (portal, blog o wiki); la extensión del enlace analizado (número de fragmentos que compone el enlace); la disposición de mapa de sitio en el enlace analizado; la visibilidad y la popularidad del portal, según Yahoo! Search ${ }^{8}$ y Google Rank; ${ }^{9}$ el uso de canales sindicados en el portal

\footnotetext{
http://public.web.cern.ch/public/

http://es.search.yahoo.com/

http://www.prchecker.info/check_page_rank.php
} 
(RSS); el uso de alertas para la recepción de mensajes y publicaciones hechas en dichos portales, por parte de sus potenciales usuarios, así como de podcast, foros, chats, y uso de Web 2.0 para divulgar contenidos del portal, tanto por éstos como por el mismo portal; el público (target) y el objetivo del contenido expuesto en el enlace analizado; y el empleo de documentos, vídeos, fotografías y enlaces de otras instituciones ajenas al Cern, entre otros aspectos medidos aquí.

Cada una de las variables consideradas para el desarrollo de este estudio permitió establecer el marco de referencia que ayudó a concretar el objetivo propuesto en este artículo, teniendo en cuenta que la mejor forma de estudiar los escenarios de divulgación científica, al interior del proyecto de estudio (Atlas), fue el abordaje transversal de los diferentes enlaces articulados al portal web principal de éste, por medio del delineamiento preliminar de un mapa de sitio que ayudase a identificar el mayor número de URL existentes para determinar el universo de estudio y la posterior muestra tomada en él.

A partir del mapa del sitio construido en diciembre de 2010, para los fines expuestos en el anterior párrafo, se logró identificar un total de 1.756 enlaces URL, que cumplen diversas funciones dentro del complejo entramado científico dentro del proyecto Atlas.

Tabla 1: Clasificación de enlaces identificados, a partir de su construcción del mapa del sitio web del proyecto Atlas hecha en diciembre de 2010

\begin{tabular}{|l|c|c|}
\hline \multicolumn{1}{|c|}{ Tipo de enlaces } & $\begin{array}{c}\text { Número de enlaces con } \\
\text { acceso abierto }\end{array}$ & $\begin{array}{c}\text { Número de portales con } \\
\text { enlaces rotos }\end{array}$ \\
\hline $\begin{array}{l}\text { Enlaces orientados a la difusión de } \\
\text { información no científica (institucio- } \\
\text { nal, oportunidades de empleo, entre } \\
\text { otras). }\end{array}$ & 316 & 44 \\
\hline Enlaces de acceso restringido & 68 & 87 \\
\hline $\begin{array}{l}\text { Enlaces orientados a la divulgación } \\
\text { científica del proyecto (exposición de } \\
\text { informes y resultados, exposición de } \\
\text { investigaciones y grupos científicos, } \\
\text { exposición de noticias y de publica- } \\
\text { ciones especializadas generadas 0 } \\
\text { relacionadas con el proyecto) }\end{array}$ & 153 & 7 \\
\hline $\begin{array}{l}\text { Enlaces institucionales que trabajan } \\
\text { en el proyecto ATLAS (proveedores, } \\
\text { por ejemplo) }\end{array}$ & & \\
\hline $\begin{array}{l}\text { Enlaces con información relacionada } \\
\text { con el traslado a las instalaciones del } \\
\text { proyecto, entre otras. }\end{array}$ & 87 & 4 \\
\hline $\begin{array}{l}\text { Enlaces con información de correos, } \\
\text { imágenes y hojas de estilo emplea- } \\
\text { dos en el proyecto }\end{array}$ & 210 & 39 \\
\hline
\end{tabular}




\begin{tabular}{|l|c|c|}
\hline $\begin{array}{l}\text { Enlaces orientados a la descarga de } \\
\text { documentación 0 archivos (instruc- } \\
\text { ciones para uso de instrumentos y } \\
\text { guías de aplicaciones web, por ejem- } \\
\text { plo) }\end{array}$ & 22 & \\
\hline Enlaces de colaboración científica & 544 & 52 \\
\hline $\begin{array}{l}\text { Enlaces que ayudan al retorno a la } \\
\text { página principal del proyecto 10 }\end{array}$ & 14 & - \\
\hline $\begin{array}{l}\text { Enlaces que no pudieron ser identi- } \\
\text { ficados ante la falta de información } \\
\text { relacionadas con estos al momento } \\
\text { de hacer el registro del sitio web }\end{array}$ & - & 32 \\
\hline
\end{tabular}

Fuente: Elaborado por el autor.

De todos los enlaces identificados se tomaron para estudio aquellos que tenían una relación directa con el tema tratado en este artículo (los escenarios de divulgación científica); es decir, 153 enlaces que pudieron ser verificados al momento de construir el mapa de sitio del proyecto, a partir de su página principal.

La muestra aplicada fue de 110 enlaces dedicados a la divulgación científica verificados con un nivel de confianza del 95\% y $\alpha=0,05$; los cuales fueron seleccionados siguiendo un procedimiento aleatorio simple en el que a cada uno de los portales se le asignó un número $\mathrm{X}$ que contribuyó a la selección de cada unidad muestral a través del uso de una tabla de números aleatorios.

\section{Resultados}

\subsection{Rasgos generales}

Para comprender mejor los rasgos que caracterizan los escenarios virtuales de divulgación científica existentes en el proyecto Atlas, ahondaremos en primer lugar en los rasgos generales de los enlaces estudiados. Al respecto, la tabla 2 nos muestra un contexto de divulgación científica marcado, en su mayoría, por portales tradicionales, en los que existe un webmaster o administrador principal, encargado de la labor de actualizar el contenido expuesto desde estos espacios; así nos ahorramos el empleo de otros tipo de escenarios virtuales, como por ejemplo Wikis y blogs, donde la labor de "actualizar" contenido está a cargo de una amplia diversidad de usuarios vinculados a éstos. 
La Tabla 2 además nos muestra cómo la labor de divulgación científica ejercida desde el proyecto Atlas es realizada mayoritariamente a través de escenarios virtuales, directamente relacionados con The European Organization for Nuclear Research (Cern). ${ }^{11}$ Ello, en vista del bajo número de enlaces analizados, cuya Url pertenecía a universidades o instituciones, afines a Atlas, al hacer parte indirecta o no a experimentos realizados en él.

Otros aspectos que pueden ser considerados a partir de los datos expuestos en la Tabla 2 son la visibilidad y la popularidad de los escenarios virtuales de divulgación científica en Atlas. En lo que se refiere a la visibilidad, la $\mu$ observada nos permite, por una parte, ubicar a nivel muy bajo la capacidad tanto en el número de enlaces recibidos como en el de indexados en cada enlace analizado, y por la otra, establecer un nivel medio de popularidad en los enlaces analizados. En el caso de los niveles de popularidad, los datos obtenidos sólo nos permiten ubicar este aspecto a nivel medio, en el 36\% de los enlaces analizados, ya que un elevado número de enlaces de nuestra muestra no permitieron arrojar datos sobre ello, como resultado de, por ejemplo: la extensión de sus Url y la falta de terminación en muchos de los enlaces estudiados de terminaciones punto.com, punto.php o punto.html.

Uno de los aspectos que nos resulta interesante es ubicar la prioridad que puede existir dentro de Atlas, al momento de llevar a cabo la generación de mecanismos de divulgación del conocimiento científico producido dentro de esta comunidad. Para ello tomamos en consideración la ubicación dentro del mapa de sitio que poseían los enlaces analizados, dividiendo la estructura de cada enlace analizado en fragmentos demarcados por una barras inclinada ( / ), la cual suele ser empleada en la programación de sitios virtuales, para establecer la jerarquía de la página mostrada por cada enlace dentro de un portal o sitio web. Como vemos en la Tabla 2, en la mayoría de los casos la ubicación jerárquica de los enlaces analizados se ubicaron en un quinto nivel, al tener $(\mu=4,95)$; es decir, los escenarios virtuales de divulgación científica dentro del proyecto Atlas parecen ubicarse a niveles muy alejados de los usuarios no habituados a éste.

En lo referido a la capacidad de los enlaces analizados a brindar la capacidad de un acceso rápido de la información publicada en éstos con el fin de aumentar la eficiencia de estos escenarios en el ejercicio de la labor de divulgación científica, la Tabla 2 nos permite ver la falta generalizada dentro de estos escenarios virtuales de mapa de sitio web en ellos lo que dificultaría la accesibilidad y aproximación del contenido publicado en ellos, para quienes no tienen un contacto asiduo o están familiarizados con este proyecto. 
Tabla 2: Rasgos generales de los enlaces analizados, a partir del mapa de sitio construido en diciembre de 2010

\begin{tabular}{|l|c|c|c|c|}
\hline & $\begin{array}{c}\text { Número de } \\
\text { enlaces } \\
\text { analizados }\end{array}$ & Mínimo & Máximo & Media \\
\hline $\begin{array}{l}\text { Tipo de enlace (Blog, Wiki, } \\
\text { Portal)a }\end{array}$ & 110 & 1 & 3 & 2,92 \\
\hline $\begin{array}{l}\text { Relación del portal con el } \\
\text { proyecto Atlas b }\end{array}$ & 110 & 1 & 8 & 1,16 \\
\hline $\begin{array}{l}\text { Popularidad del portal, según } \\
\text { GoogleRank }\end{array}$ & 40 c & 0 & 723 & 10,07 \\
\hline $\begin{array}{l}\text { Enlaces indexados Yahoo! } \\
\text { Search }\end{array}$ & 108 & 0 & 541 & 13,98 \\
\hline $\begin{array}{l}\text { Enlaces recibidos Yahoo! } \\
\text { Search }\end{array}$ & 106 & 1 & 8 & 4,95 \\
\hline $\begin{array}{l}\text { Extensión del enlace } \\
\text { analizado (número de } \\
\text { fragmentos de la Url) }\end{array}$ & 110 & 2 & 2 & 2,00 \\
\hline $\begin{array}{l}\text { Disposición de mapa de } \\
\text { sitio Webb }\end{array}$ & 110 & & & \\
\hline
\end{tabular}

Fuente: Elaborado por el autor.

Nota: a $1=$ Blog; $2=$ Wiki; $3=$ Portal.

b $1=\mathrm{Si} ; 2=$ No.

c 70 enlaces no arrojaron datos durante la medición.

\subsection{Contenidos publicados}

A nivel interno los datos obtenidos al analizar los enlaces de la muestra (tabla 3) nos permiten ver el alto nivel de especialización al que se orientan los escenarios virtuales de divulgación científica, al interior del proyecto Atlas. Esto se debe a la marcada orientación de sus contenidos hacia científicos y estudiantes de áreas disciplinares afines, directamente o no vinculados con esta comunidad, y no tanto a un público más externo, conformado por otras comunidades científicas y de estudiantes, como son los periodistas especializados o personas interesadas en las temáticas que no provienen de disciplinas afines. Además, podemos ver que la mayoría de los enlaces empleados para la divulgación científica del conocimiento generado en este proyecto, tomado como caso de estudio, se orientaban, de forma prioritaria, a la exposición de informes y resultados de investigación; dejando la exposición general de investigaciones, grupos científicos conformados en él, así como la divulgación de publicaciones especializadas, relacionadas o generadas en su interior, y la exposición de noticias especializadas, en un segundo plano u orden de prioridad. 
Tabla 3: Rasgos que caracterizan los contenidos publicados en los escenarios virtuales de divulgación científica del proyecto Atlas, analizado en diciembre de 2010

\begin{tabular}{|l|c|c|c|c|}
\hline & $\begin{array}{c}\text { Número } \\
\text { de enlaces } \\
\text { analizados }\end{array}$ & Mínimo & Máximo & Media \\
\hline Público destinatario del portala & 110 & 1 & 2 & 1,15 \\
\hline Objetivo del portalb & 110 & 1 & 4 & 2,33 \\
\hline $\begin{array}{l}\text { Acceso abierto a cualquier sitio } \\
\text { del portalc }\end{array}$ & 110 & 1 & 2 & 1,02 \\
\hline $\begin{array}{l}\text { ldentificación de autor(es) de la } \\
\text { información publicada en el portalc }\end{array}$ & 110 & 1 & 2 & 1,30 \\
\hline $\begin{array}{l}\text { Referencia de enlaces de otras web } \\
\text { ajenas a Atlasc }\end{array}$ & 110 & 1 & 2 & 1,82 \\
\hline Empleo de vídeos en el portalc & 110 & 1 & 2 & 1,91 \\
\hline $\begin{array}{l}\text { Empleo de podcast (audios) en } \\
\text { el portalc }\end{array}$ & 110 & 1 & 2 & 1,98 \\
\hline $\begin{array}{l}\text { Empleo de fotografía o infografía en } \\
\text { el portalc }\end{array}$ & 110 & 1 & 2 & 1,36 \\
\hline Empleo de documentos publicadosc & 110 & 1 & 2 & 1,14 \\
\hline
\end{tabular}

Fuente: Elaborado por el autor.

Nota: a 1=Público experto (científicos y estudiantes de disciplinas afines); $2=$ Público general (científicos y estudiantes, periodistas especializados y personas interesadas, ajenos a las disciplinas)

b 1=Exposición de investigaciones y grupos científicos; $2=$ Exposición de informes y resultados de investigación; 3 =Exposición de noticias especializadas; $4=$ Exposición de publicaciones especializadas.

c $1=\mathrm{Si} ; 2=$ No.

En lo que se refiere al acceso a la información publicada en los enlaces virtuales orientados a la divulgación científica dentro del proyecto Atlas, los datos obtenidos de la muestra (Tabla 3), nos permiten apreciar la presencia generalizada en estos escenarios del "acceso libre" de sus contenidos; es decir, en general, los espacios virtuales dedicados a la divulgación científica en este proyecto no establecen restricciones para el acceso a determinadas zonas de los contenidos expuestos en los enlaces analizados. Así mismo se puede ver que en la mayoría de los enlaces también se identifican los autores de los contenidos publicados, lo cual ayuda al usuario a relacionar a los grupos de investigación vinculados a este proyecto, con determinadas zonas o enlaces dedicados a la divulgación científica, por medio del contenido expuesto en ellos.

Otro de los aspectos vistos en la Tabla 3, que caracterizan los escenarios virtuales de divulgación científica en Atlas, es el bajo nivel de empleo de referencias ajenas al proyecto; en la mayoría de los casos provenientes de instituciones afines o vinculadas a investigaciones ejecutadas en su interior. Esto nos muestra que el marco de divulgación científica está caracterizado en Atlas por la divulgación científica "propia” y no por el ejercicio de esta labor de 
forma horizontal; es decir tratando de relacionar los conocimientos elaborados por otros.

Uno de los rasgos que caracterizan los escenarios virtuales en la actualidad es la especialización de los formatos de divulgación de los contenidos generados por sus autores, (García, 2004). Además de los contenidos divulgados dentro de los formatos html u otros empleados en cada uno de los enlaces analizados a través de hipervínculos o información directamente publicada en estos, los resultados expuestos en la Tabla 3 nos muestran una reproducción del modelo tradicional de comunicación tradicional en estos escenarios de divulgación científica (Díaz-Noci, 1997), ya que la exposición de sus contenidos se hace por medio de enlaces que permiten la apertura o descarga de documentación elaborada por los científicos, y a través de imágenes o fotografías de las investigaciones y resultados obtenidos por éstos; dejando en casos puntuales, el uso de videos y podcast (audios). Por tanto las posibilidades de personalizar los diferentes escenarios de divulgación científica analizados en Atlas, de acuerdo con un mayor volumen de usuarios muy pequeños e individuales que podrían estar interesados en acceder a esta información, difícilmente pueden ser atendidas bajo el escenario actual, ya que la mayor parte de la información expuesta en estos escenarios resulta altamente especializada y tecnificada y no reproducida en múltiples formatos que ayuden a "acercar" el conocimiento dispuesto en este proyecto a colectivos ajenos a él.

\subsection{Herramientas de interacción y personalización de la información}

Hume (1996) y Briggs (2007), entre otros; han dado cuenta de los avances que han generado las TIC en el cambio del discurso unidireccional de los escenarios virtuales; así como del auge de una nueva generación de usuarios en estos contextos, más participativos, en donde la habilitación de procesos de intercambios y feedback resultan claves, en tanto que permiten adquirir un rol de agente de divulgación de la información expuesta en estos enlaces, por medio de sus canales de comunicación o de otros abiertos por el medio original, generador del contenido.

Los resultados obtenidos (Tabla 4) nos permiten señalar el bajo nivel de empleo de herramientas y de interacción y personalización de la información en los escenarios virtuales de divulgación científica analizados desde el proyecto Atlas.

Como bien han expuesto Martínez, Peñalver y Salamanca (2007), Franco (2007), Fumero y Roca (2007) y Codina (2009), entre otros investigadores, el desarrollo de las Web 2.0 abren un nuevo escenario en el que se tiene la posibilidad 
de ampliar las comunidades alrededor de los escenarios de comunicación virtuales existentes, a favor de la apertura de nuevos mecanismos de atracción, fidelización y difusión de contenidos, más allá de los enlaces dispuestos para tal fin. Este hecho también puede ser aplicado en los escenarios virtuales de divulgación científica, en nuestro caso los existentes al interior del proyecto Atlas.

Los resultados de este trabajo nos muestran un muy bajo nivel de uso de las Web 2.0 dentro del proyecto tomado como caso de estudio, bien sea permitiéndole a quienes acceden a los enlaces analizados agregar y compartir contenido, o divulgar desde sus portales los contenidos desarrollados por los miembros de la comunidad científica participante en Atlas. Los resultados expuestos en la tabla 4, confirmarían lo antes señalado, lo que quizá reduciría, gracias al auge de las Web 2.0 y las herramientas de participación de usuarios en las dinámicas de acción de los portales web existentes; es decir, las oportunidades de aumentar el impacto divulgativo de los contenidos generados dentro de la comunidad científica estudiada (Atlas).

Tabla 4: Herramientas de interacción y personalización de la información en los escenarios virtuales de divulgación científica del proyecto Atlas, analizado en diciembre de 2010

\begin{tabular}{|l|c|c|c|c|}
\hline & $\begin{array}{c}\text { Número de } \\
\text { enlaces } \\
\text { analizados }\end{array}$ & Mínimo & Máximo & Media \\
\hline $\begin{array}{l}\text { Uso de canales sindicados en } \\
\text { el portala }\end{array}$ & 110 & 1 & 2 & 1,95 \\
\hline $\begin{array}{l}\text { Uso de servicio de alertas en } \\
\text { el portala }\end{array}$ & 110 & 1 & 2 & 1,97 \\
\hline $\begin{array}{l}\text { Posibilidad de introducir comentarios } \\
\text { por visitantes en el portala }\end{array}$ & 110 & 1 & 2 & 1,95 \\
\hline $\begin{array}{l}\text { Posibilidad de envío de contenidos } \\
\text { del portal por emaila }\end{array}$ & 110 & 1 & 2 & 2,00 \\
\hline $\begin{array}{l}\text { Disposición de foros de } \\
\text { discusión en el portala }\end{array}$ & 110 & 2 & 2 & 2,00 \\
\hline Disposición de chats en el portala & 110 & 1 & 2 & 1,95 \\
\hline $\begin{array}{l}\text { Disposición de herramientas Web } \\
\text { 2.0 para compartir contenidos del } \\
\text { portala }\end{array}$ & 110 & 1 & 2 & 1,95 \\
\hline $\begin{array}{l}\text { Disposición de canal Web 2.0 para } \\
\text { divulgación del contenido del portala }\end{array}$ & & 2 & & \\
\hline
\end{tabular}

Fuente: Elaborado por el autor.

Nota: a $1=\mathrm{Si} ; 2=$ No. 


\section{Conclusiones}

Como expusimos al comienzo de este artículo nos encontramos en un contexto actual donde las comunidades científicas cuentan con nuevas herramientas para publicar y compartir el conocimiento generado en estos espacios académicos.

En el caso del proyecto Atlas podemos establecer un contexto de colaboración a partir de los escenarios virtuales de divulgación científica analizados, donde el aprovechamiento de estos contextos analizados sigue presentando limitaciones, pese a la compleja red de micro-portales dispuestos dentro de este proyecto, ya que los datos obtenidos nos permiten no sólo apreciar la adhesión de políticas de libre acceso del conocimiento producido, extendido al interior de esta comunidad, sino también observar las limitaciones existentes en torno a otros tipos de oportunidades que ofrece Internet, al momento de aumentar el impacto del trabajo realizado en este proyecto, en lo que se refiere a comunidades, científicas o no, no vinculadas al trabajo realizado. Esto quizá se deba al poco uso de canales virtuales más participativos, el empleo de modelos de comunicación digital tradicionales (imágenes y documentos), y al bajo nivel de uso de las herramientas de interacción y personalización de la información dispuestas en los escenarios virtuales de divulgación científica analizados. Ello, pese a que según la clasificación propuesta por David (2006), el proyecto Atlas se caracteriza por tener diferentes niveles de aplicabilidad de la e-ciencia con base en los diferentes micro-sitios o portales entrelazados en él; sin que ello esté generando una promoción elevada de mecanismos de aparición de super-usuarios (Fumero y Roca, 2007), encargados de producir y consumir los contenidos, informaciones y conocimientos generados al interior de este proyecto.

En el caso del proyecto Atlas, pese a un intento por aumentar las rutas de comunicación, de difusión y de visibilidad de la producción intelectual efectuada al interior de este proyecto con otros usuarios, a través de Internet, (Russel, 2001; Pila, 2009; Gómez y Arias, 2002), el aprovechamiento de estos contextos virtuales aún sigue estando limitado, en vista del poco empleo de las capacidades actuales de las TIC y la alta presencia de material expuesto codificado, no dirigido a comunidades no generadoras de estos saberes, no afines a disciplinas vinculadas con este caso de estudio. Por tanto podemos decir que el nivel donde parece encontrarse los escenarios de divulgación científica en Atlas es medio, si consideramos lo expuesto por Lugo (2004) y otros investigadores referenciados en este artículo, en torno al cambio de parámetros de diseminación y visibilidad de la información o contenidos desarrollados por esta importante comunidad científica. 
En cuanto al tipo de divulgación científica generada desde el proyecto Atlas, los resultados nos llevan a señalar que, de acuerdo con la clasificación expuesta al comienzo de este artículo, a partir de la propuesta de Fernández (1999) y León (1999), la modalidad más próxima a lo visto a partir de los datos es la realizada por expertos para expertos. Ello en vista de que en la mayoría de los enlaces analizados, los contenidos elaborados están siendo dirigidos a una audiencia (usuarios) que forma parte del mismo colectivo científico-investigador. Este hecho nos lleva a recordar lo expuesto por Estrada (2003), en lo que se refiere a la capacidad que debe tener la comunicación aplicada en la divulgación científica para darle acceso al conocimiento a un público ajeno a éste, lo que llevaría a señalar que, al menos en el caso del proyecto Atlas, las posibilidades de apropiación significativas del saber generado en él, resultan limitadas, solo a expertos afines a las disciplinas que hacen vida en ellas, lo que pudiese traducirse en la presencia actual de barreras que pueden estar impidiendo la apropiación significativa de estos saberes a otros actores de nuestras sociedades.

Con base en lo expuesto aquí y en los resultados obtenidos en este artículo resulta que:

- A nivel del carácter divulgativo de los espacios virtuales analizados, la exposición de contenidos dentro del proyecto Atlas, en vista del elevado nivel de codificación de los contenidos existentes en dichos contextos, se orienta más a promover espacios de intercambio entre especialistas del mismo campo interdisciplinar.

- La promoción de la e-ciencia desde los escenarios analizados del proyecto Atlas, a través del aprovechamiento de las posibilidades dialógicas, colaborativas y participativas de las Web 2.0, si bien resulta paradigmática, en vista del número de investigadores, instituciones y países interconectados en torno a una misma temática de interés, aún parece ubicarse en el contexto de la promesa y no en la concreción efectiva (real) de las potencialidades brindadas por las herramientas 2.0 para desarrollar nuevos horizontes formativos e investigativos. Ello, en vista de: 1) el carácter centrípeto que pareciera regir el uso de los recursos de colaboración dispuestos en él, orientados hacia los miembros de dicha comunidad; 2) la baja aplicabilidad de blogs, zonas de comentarios, wikis y chats abiertos a usuarios (miembros o no) interesados en los temas abordados en este proyecto; y 3 ) el sentido que se le da al uso de las herramientas 2.0 a nivel externo, donde pareciera que el principal objetivo de éstos se centrará en la búsqueda de mecanismos que garanticen la promoción del proyecto Atlas, desde otros canales personales o pertenecientes a otras comunidades afines. 
- Si bien el proyecto Atlas, a través de los escenarios virtuales analizados promueve el desarrollo de comunidades de aprendizaje alrededor del tema abordado en él, lo expuesto por Castaño et al (2008) y Tejedor et al (2011), en relación con la aparición de nuevas oportunidades de aprendizaje colaborativo, la generación de nuevos contextos de reflexión y aplicación de prácticas de validación; de contrastes y filtrado de datos y contenidos dispuestos desde Internet, y el fomento de una cultura de la e-ciencia entre quienes están fuera de los miembros del proyecto Atlas resulta, por desgracia, bajo. Por tanto, en mi opinión, se estarían aprovechando poco los escenarios virtuales analizados orientados a fortalecer dinámicas que ayuden a otras comunidades científicas, ajenas o relacionadas indirectamente al tema tratado en él, como serían los docentes de institutos de educación secundaria, y los grupos de investigación e investigadores de otros campos disciplinares, por ejemplo, a avanzar hacia lo antes expuesto, por medio del contacto directo con este proyecto.

\section{Bibliografía}

Agenjo, Xavier, "La Hipótesis de Ortega", en Revista de estudios orteguianos, 2002, núm. 5, pp. 103-110.

Bailon-Moreno, Rafael et al, "The Pulsing Structure of Science: Ortega Y Gasset, Saint Matthew, Fractality and Transfractality”, en Scientometrics, 2007, vol. 71, núm. 1, pp. 3-24.

Biro, Susana, Miradas Desde Afuera: Investigacion Sobre Divulgacion, Ciudad de México: Universidad Nacional Autónoma de México, 2007.

Briggs, Mark, Periodismo 2.0. Una guía de alfabetización digital, 2007, http://knightcenter.utexas.edu/Periodismo_20.pdf.

Cabeza-Clavijo, Alvaro; Torres-Salinas, Daniel; Delgado-López-Cozar, Emilio, "Ciencia 2.0: catálogo de herramientas e implicaciones para la actividad investigadorä, en El Profesional de la Información, 2009, vol. 18, núm. 1, pp. 72-80.

Codina, Lluís, Metodología de Análisis y Evaluación de Recursos Digitales en Línea, 2006, http://www.lluiscodina.com/metodos/metodos2006.doc.

Codina, Lluís, "Ciencia 2.0: Redes sociales y aplicaciones en línea para académicos", Hipertext.net, 2009, núm. 7 http://www.hipertext.net

David, Paul, "Toward a cyberinfrastructure for enhanced scientific collaboration: providing its "soft" foundations may be the hardest part", en Kahin, Brian; Foray, Dominique, (Eds.), Advancing Knowledge and the Knowledge Economy, Cambridge: MIT Press, 2006, pp. 431-453. 
Díaz-Noci, Javier, “'Tendencias del periodismo electrónico. Una aproximación a la investigación sobre medios de comunicación en internet", en Zer, 1997, núm. 2. http://www.ehu.es/zzer/ zer2/6artdiaz.html.

Estrada, Luis, La Divulgacion de la Ciencia: Educacion, Apostolado O...? Ciudad de México: Universidad Nacional Autónoma de México, 2003.

Fernández, Francisco, Traducción y retórica contrastiva a proposito de la traducción de textos de divulgación cientifica del inglés al español, Santiago de Compostela: Univ Santiago de Compostela, 1999.

Franco, Guillermo, Cómo escribir para la Web. Bases para la construcción y discusión de manuales de redacción online, 2007, http:// knightcenter.utexas.edu/como_web.php.

Fumero, Antonio; Roca, Genís, Web 2.0. Madrid, España: Fundación Orange, 2007.

García, Elvira, "La especialización en la era de internet", en Fernández-Del-Moral, Javier (Coord.), Periodismo especializado, Madrid: Ariel, 2004, pp. 195-218.

Glasner, Paul, "From community to 'collaboratory'? The Human Genome Project and the changing culture of science", en Science and Public Policy, 1996, v. 23, núm. 2, pp. 109-116.

Gómez, N. y Arias, O, "El cambio de paradigma en la comunicación científica”. Información, cultura y sociedad, 2002, núm. 6, pp. 93. 102, http://www.scielo.org.ar/scielo.php?script=sci_arttext\&pid $=$ S1851-17402002000100007\&lng $=$ en $\& n r m=$ iso \& $\operatorname{lng}=$ es

Harnad, Stefan, "The self-archiving initiative", en Nature, 2001, núm. 410, pp. 1024-1025.

Hume, Ellen, "The new paradigm for news", en Annals of the American Academy of Political and Social Science, 1996, v. 546, núm. 1, pp. 141-153.

León, Bienvenido, El documental de divulgación cientifica, Barcelona: Editorial Paidós, 1999.

Lugo, Margarita, "Evaluación de recursos digitales: análisis de algunos parametros", en Revista Digital Universitaria, 2004. http:// www.revista.unam.mx/vol.5/num6/art38/art38.htm.

López, Maricela; C ordero, Graciela, "Un intento por definir las características generales de las revistas académicas electrónicas", en Razón y Palabra, 2005, núm. 31. http://www.razonypalabra.org. $\mathrm{mx} /$ libros/libros/caracrevelec.pdf.

Nafría, Ismael, Web 2.0. El usuario, el Nuevo rey de internet, Barcelona: Gestión, 2000, 2007.

O'Reilly, Tim, "What is Web 2.0. Desing Patterns and Business Models for Next Generation of Software", en O'Reilly.com, 2005, http://oreilly.com/web2/archive/what-is-web-20.html

Pila, Justine, "Authorship and e-Science: Balancing Epistemological Trust and Skepticism in the Digital Environment", en Social Epistemology, 2009, núm. 23, pp. 1-24. 
Rodríguez-Martínez, Ruth; Codina, Lluís; Pedraza-Jiménez, Rafael, "Cibermedios y web 2.0: modelo de análisis y resultados de aplicación", en El Profesional de la Información, 2010, vol. 19, núm. 1, pp. 35-44.

Rubio, Julio, "Lenguaje y Comunicación en la Ciencia", en Razón y Palabra, 1998, http://www.razonypalabra.org.mx/anteriores/n12/ leng12.html.

Russell, Jane, "La comunicación científica a comienzos del siglo XXI", en Organización de Estados Iberoamericanos, 2001, http://www. oei.es/salactsi/rusell.pdf.

Said-Hung, Elias; Arcila-Calderón, Carlos; Méndez-Barraza, Jorge, "El desarrollo de los cibermedios en Colombia", en El Profesional de la Información, 2011, vol. 20, núm. 1, pp. 47-53.

Tejedor, Santiago; Baena, Glòria; Sanz, Mireia; Ortuño, Xavier, "La Web 2.0 en Iberoamérica: Estrategias para una alfabetización ciberperiodística”, en Consejo Nacional de Televisión de Chile, 2011, http://www.cntv.cl/prontus_cntv/site/edic/base/port/inicio.ht$\mathrm{ml}$

Toffler, Alvin, La Tercera Ola, Bogotá: Plaza \& Janes Editores, 1980.

U.S Departament of Energy, "The sensitive giant. At CERN, ATLAS effort emphasizing people skills", en U.S. Department of Energy. Research News, s.f., http://www.eurekalert.org/features/doe/200403/dnal-tsg032604.php. 
